\title{
Doing the month in a Taiwanese postpartum nursing center: An ethnographic study
}

\author{
Yeh, Y., St John, W., \& Venturato, L. (2014). Doing the month in a Taiwanese postpartum nursing \\ center: An ethnographic study. Nursing and Health Sciences, 16(3), 343-351. \\ DOI: http://onlinelibrary.wiley.com/doi/10.1111/nhs.12110/pdf
}

\begin{abstract}
Traditionally, Chinese postpartum women conducted postpartum ritual practices, called 'doing the month' at home. Today, many Taiwanese women undertake this ritual in postpartum nursing centers. This ethnographic study investigated how the traditional practices are being transformed in relation to contemporary health care in Taiwan. Observations were carried out in a large post-partum center attached to a major hospital in Taipei for six months, and twenty seven postpartum women were interviewed. Doing the month practices are being reshaped by being relocated from the home to a health care setting. Midwives took on roles traditionally taken by family members, which impacted on family roles and relationships. Some postpartum practices related to diet, hygiene, social activity and social activity were maintained, based on traditional explanations. However, many were modified or challenged based on explanations from contemporary scientific knowledge. This study informs culturally appropriate postpartum care and support for women with traditional and contemporary cultural beliefs and attitudes to doing the month in a range of healthcare contexts.
\end{abstract}

\section{Key words}

Traditional Chinese culture, cultural values, ethnography, postpartum care, Taiwan 


\section{INTRODUCTION}

Childbirth is usually a time of transition and social celebration, and often initiates adjustments in cultural responsibilities (Steinberg, 1996). In numerous societies, including Chinese societies, postpartum care is influenced by both traditional beliefs and contemporary health-care practices (Lauderdale, 1999; Posmontier \& Horowitz, 2004). 'Doing the month' is a 30-day Chinese postpartum ritual involving physical and social prescriptions and taboos. According to Chinese folk and classical medicine, postpartum women are in a state of extreme yin and yang imbalance, which may result in weakness and vulnerability (Chen, 2001). This type of weakness is regarded as dangerous to physiological health and makes new mothers vulnerable to "heat" or "cold", which may cause health problems such as dizziness, headaches, backache, and arthralgia during the month or in later years (Pillsbury, 1978) . Furthermore, during this time, wind and water are considered dangerous because they may enter the body through skin pores and cause tumors, weakness and arthritis (Liu et al., 2012). Therefore, Chinese women are advised to follow a particular diet and health-care practices.

To do the month, postpartum mothers are advised to confine themselves, rest completely, and avoid bathing or washing their hair to prevent contact with yin, such as cold air, wind and water. As a result, postpartum women are typically confined to the home, stay in bed, windows are typically kept closed; and air conditioning is avoided, even in the hot, humid Taiwanese summer. In addition, postpartum women are encouraged to eat 'hot' or yang foods, such as rice wine, food cooked with ginger and sesame oil, kidney, liver, chicken and eggs for four weeks. "Cold" or yin foods, such as turnips, Chinese cabbage, bamboo shoots, and leafy green vegetables are considered to weaken a postpartum woman's health and should be avoided (Liu et al., 2012; Strand et al., 2009). Postpartum women are considered to be polluted during the 30-day postpartum period. In addition, the woman cannot have sex with her husband during this time, because it is believed to bring misfortune (Pillsbury, 1978). These rituals, together with restrictions on social activities are observed to provide a new mother with time to rest, regain strength and learn to care for the baby (Posmontier \& Horowitz, 2004).

The international literature shows that in many countries many new mothers spend relatively little time in hospital after childbirth, but feel a need for additional support, guidance, and assurance from healthcare professionals (Forster et al., 2008; Hung et al., 2010; Martin et al., 2013). Postpartum mothers' experiences of being discharged from maternity hospitals have been described as unpredictable and stressful (Howell, 2010; Martin et al., 2013), especially for first-time mothers (Forster et al., 2008; Ngai \& Chan, 2012; Ohashi \& Asanorn, 2012). In Taiwan, childbirth fundamentally changes and increases the complexity of a new mother's life, not only because of increased demands, conflict and stress (Gaoa et al., 2013), but also because of their inexperience with following the ritual practices of doing the month within the realities of contemporary society (Hung et al., 2012; Liu-Chiang, 1995; Liu et al., 2006). Traditional ritual practices that contributed to social order in the past are now being challenged and transformed in response to social changes, such as changing family structures, 
women's status, and health-care systems. These social changes also affect the ritual practices of doing the month. In Taiwan, the national healthcare system no longer provides postpartum home visits, and other postpartum-related services are quite limited (Hung et al., 2012). Therefore, numerous Taiwanese women now follow doing the month in postpartum nursing centers (PNC) after being discharged from maternity hospitals.

PNCs represent themselves as providing new mothers with a feasible alternative to doing the month at home, and are an increasingly popular choice for affluent contemporary women (Chen, 2011; Hung et al., 2010). New mothers are admitted to PNCs after being discharged from maternity hospitals. Most PNCs are staffed by physicians and midwives who are responsible for caring for the baby and facilitating mothers' recovery, suggesting that it is possible to combine contemporary societal values and modern health-care delivery practices with traditional ritual practices (Hung et al., 2010).

The services provided by PNCs indicate the development of a new type of health industry that differs from early postpartum care and post-discharge postpartum care. Today, 166 PNCs are operating in Taiwan (National Statistics ROC Taiwan, 2013). Thus, doing the month practices are now being revived and transferred outside the family by contemporary Taiwanese women. However, little is known about how doing the month practices are being transformed in relation to contemporary health care in Taiwan today.

\section{Aim}

The purpose of this study was to explore how the traditional Chinese postpartum ritual of doing the month is being reshaped for new mothers in a contemporary healthcare context at a PNC in Taiwan.

\section{METHODS}

\section{Design}

An ethnographic approach was used for this study. Ethnography reveals the particularities of cultures and rituals (Denzin, 1996; Munhall, 2007), enables cultural knowledge to be elicited within a specific community or setting, in this case a PNC, by observing events, listening to conversations and asking questions (Hammersley \& Atkinson, 2007).

\section{Setting}

The PNC selected for this study was located in Taipei City, where traditional cultural practices and cosmopolitan values and practices influence each other. The demography of women living in Taipei City reflects the changing position of women in Taiwanese society. Observation and interview data were collected by the first author over a period of nine months in a 36-bed PNC that is affiliated with a hospital. The PNC was unfamiliar territory for the first author, and she had no prior connection to the staff or the PNC. This allowed her to act as a 'cultural stranger' (Maso, 2001) who does not make assumptions about the social or cultural group being studied and allowed her to maintain a professional distance. At the time of the fieldwork, the PNC was staffed by medical, nursing and allied 
health practitioners. The ratio of midwives to babies and mothers was 1:12-15. Daily costs and charges in the PNC were at $\$ 120$ to $\$ 140$ USD ( $=\$ 3600 \sim \$ 200$ NTD), which is expensive for most Taiwanese families.

\section{Participants}

The main criterion for participant selection for interviews was that participants were first-time mothers who had given birth to healthy newborns and were seeking to maintain the traditional practices of doing the month by attending the PNC. Additional selection criteria included the ability to speak Chinese and willingness to participate in the study. All participants were assured of confidentiality and given the option of withdrawing from the study at any time. A total of 27 first-time mothers at the PNC were recruited for formal semi-structured interviews, to satisfy the principle of saturation (Hammersley \& Atkinson, 2007).

\section{Data Collection}

Data collection in ethnographic fieldwork requires collecting data in a natural setting from a variety of data sources such as observation and individual interviews with new mothers, informal conversations with staff and visitors. Martin (1992, p. 7) argued that inclusion of multiple voices was desirable, because different members of a culture have different opinions. Semi-structured interviews and key informant interviews were conducted to uncover the new mothers' day-to-day activities related to doing the month. The first author usually conducted the one-on-one interviews in the participant's room, which was quiet and private. Each interview lasted between 40 and 80 minutes. All interviews were audio-recorded (with interviewees' permission) for transcription and analysis purposes. Each interview was transcribed and translated into English by the first author. A conversation guide was prepared that aimed to encourage participants to describe their stories, experiences and attitudes toward the ritual practices of doing the month (Table 1). In addition, a brief demographic profile was obtained to describe the participant group. (Table 2)

During the observation period, the researcher was consistently present in the PNC, but followed a flexible time schedule (Bernard, 2011). In addition to the formal interviews with 27 new mothers, observations included the daily activities of the PNC, the staff, new mothers, and visitors, the new mothers' educational sessions, as well as informal conversations with new mothers and visitors. The observations and interviews provided a systematic method for observing, describing, documenting and capturing the daily activities and nuances (Leininger, 1991) related to the practice of doing the month in the PNC.

\section{Ethical Considerations}

This study was approved by the Griffith University Human Research Ethics Committee (HREC) in Australia and the participating PNC in Taiwan. Throughout the study, the researchers fully considered participants' human rights. The purpose of the study was explained, and confidentiality was assured. To protect the identity of the participants and the agency, the agency has not been identified and data have been de-identified before reporting results. 


\section{Rigor}

To enhance the trustworthiness of the findings, credibility, dependability and confirmability were established (Lincoln \& Guba, 1985). The first author is an experienced registered midwife and has been an obstetrics educator for more than 10 years. The researcher's background and experience enabled her to build trust with participants when discussing their daily lives related to doing the month. Credibility is achieved by prolonged engagement and member-checking with the participants. Furthermore, verbatim quotations are used during presentation of findings to allow readers to judge the veracity of the work (Lincoln \& Guba, 1985).

\section{Data Analysis}

Although analytical approaches followed in ethnographic studies are often debated, the crucial consideration is that researchers must be able to describe people and their culture (Hammersley \& Atkinson, 2007). By locating the study at a PNC, it was possible to understand how doing the month was performed in this context. A multi-step analysis technique developed by Creswell (Creswell, 2003) was used, which involves a cyclical process of data collection and verification at every step of the inquiry to construct a solid product. Data were manually analyzed by underlining key words in the transcripts, decreasing code phrases, shaping concepts, grouping concepts into categories, and classifying the categories. These steps facilitated a rigorous and transparent analysis process (Munhall, 2007).and proved to be useful throughout the complex processes of identifying, exploring, developing, and enriching the interpretation of data. The researcher's prolonged engagement and familiarity with the target culture facilitated her sense of the data, allowing her to become a storyteller about the processes of doing the month in this PNC context.

\section{RESULTS}

Demographics details of the 27 new mother interview participants are presented in Table 2. The new mothers were aged between 26 and 38 years old, with a mean age of 32 years. All participants were married ( $\bar{x}=3.4$ years) and the majority $(81 \%, n=22)$ lived with only their husbands. Education levels ranged from junior college to doctorate levels. The following section outlines categories that emerged from the interviews: dietary practices; restricted physical activities; restricted hygiene practices; and social proscriptions (Table 3). The reasons for doing the month at a PNC is overviewed, because they provide an important context for the practices.

\section{Reasons for Performing Doing the Month at a Postpartum Nursing Center}

Participants usually made the decision to do the month at a PNC after discussing it with their husbands, friends, and family, often making their final decision after consulting with both their mother and mother-in-law. Participants had generally been brought up to believe that adherence to this ritual was physically and psychologically appropriate, and that it prevents them from experiencing illnesses in the future. Participants' reasons for deciding to perform doing the month at the PNC included:

1) respect for the traditional ritual of doing the month and a belief that the practices would nurture their physical recovery. For instance, participants stated that: "[I will be] able to rest properly [at the PNC]"; 
2) to learn childcare skills;

3) to avoid inter-generational conflicts about how to perform doing the month, particularly in relation to hygiene practices;

4) convenience and autonomy and an ability to make their own decisions. In particular, participants identified that doing the month at a PNC "saves trouble," and is "more comfortable and involves fewer restrictions";

5) lack of knowledge and familiarity with complex doing the month procedures, for example: "[I] don't know how to prepare doing the month meals"; and

6) having limited support at home. It was clear that most of the participants had been working and lived in a nuclear family, which meant that they lacked the necessary family support to perform doing the month.

\section{Dietary Practices: Yang Foods}

Dietary practices at the PNC were generally performed in accordance with contemporary nutritional principles, which were introduced by a nutritionist during an educational program. Several foods traditionally eaten during doing the month were provided. One new mother mentioned she took advantage of foods offered at the PNC that were consistent with traditional practices, and indicated that she felt optimistic about the effect on her wellbeing:

"I know I cannot eat something cold. I have sesame oil chicken and liver..., I believe doing the month well would help adjust my physical constitution".

At the PNC, most traditional dietary practices related to the concept of well-balanced nutrition and yang foods were maintained. The participants asserted that they needed nutrient-rich foods to maintain their health and to produce breast milk for their babies. Several participants identified that it was appropriate to eat high-quality food that contains large amounts of protein, iron, and lipid-soluble vitamins, including meats, fish, chicken and internal organ such as pig's liver in their diet.

\section{Dietary Practices: Yin Food}

Traditionally, postpartum women were advised to avoid yin foods, such as vegetables and fruit. Most of the new mothers at the PNC prescribed to food taboos. Although some vegetables and fruit were considered "cold" in nature, the women generally believed that vegetables and fruit were needed to ensure a balanced diet that included a comprehensive range of nutrients. They believed that it was acceptable to select "neutral" vegetables and fruit and to avoid vegetables and fruits that are "cold". For example, one woman knew that she was supposed to avoid eating "cold" and "raw" foods. She preferred to select her food based on this knowledge, and she combined the traditional requirements with contemporary nutritional concepts:

"Vegetables and fruit are good for my health, you know...like a balanced diet. So I chose vegetables and fruit that are 'neutral' such as cauliflower, grapes, strawberries, and apples."

Several new mothers preferred to follow the professional health care staffs' instructions related to nutrition, which were mainly constructed in accordance with contemporary nutritional principles and 
evidence.

\section{Restricted Physical Activities}

All participants in this study maintained the traditional practice of not performing household duties, and most rested in bed the majority of the time. A major reason provided for being at the PNC was to avoid the physical exertion of household duties and to gain sufficient rest, regain energy, and maintain good health. One woman stated:

"If I practiced doing the month at home, I would surely do something [housework] and walk around. As a result, it would be even harder for me to have good rest. To practice doing the month here makes it easier for me to focus on myself...I hope to take advantage of doing the month to take good care of myself."

Most of the new mothers adhered to the ancient taboo against going out socially because of the belief that they were in a weakened condition after childbirth, and the majority of the new mothers never participated in outdoor activities. They stayed inside the PNC for the entire month, believing that the ancestors' prescriptions were sensible, and that the ritual would ensure their future health. Physical activities were therefore limited, despite current scientific knowledge demonstrating the importance of physical activity and encouragement from the PNC staff to partake in postpartum exercise classes.

\section{Restricted Hygiene Practices}

Traditionally, new mothers are not encouraged to take baths or wash their hair for 30 days. However, several participants washed and bathed at the PNC, even though they knew that bathing and washing were not culturally permitted during this period. They neglected to follow these restricted hygiene practices because of personal preferences and a sense that not washing was dirty. One woman mentioned that:

"If I did not wash, I would feel dirty and sticky with breast milk. I think I should keep clean, because I need to hold my baby and breastfeed."

Rigid adherence to the prohibition of washing and bathing was difficult from a hygiene and comfort perspective. Although some of the new mothers did not bathe or wash their hair during the doing the month period, most challenged or modified these practices, arguing that contemporary facilities and scientific knowledge inform contemporary hygiene and comfort practices. In an interesting interaction with technology that was not available to previous generations, some new mothers washed their hair and then immediately used a radiant heat hair dryer. One woman clarified that:

"The taboo of not washing hair originated from rural ancient China, where there was a poor living standard...I washed my hair and dried it with a hair dryer straight away. It is not necessary to follow the taboo [restricted washing] strictly."

A participant indicated that she had trouble adhering to the rules and felt extremely uncomfortable without washing and bathing. She believed that she needed to maintain contemporary standards of hygiene:

"I believe I would feel terrible if I didn't bathe, and I wouldn't like to practice doing the month with such discomfort. Because I have spent money on doing the month, I would surely hope to do it in my way." 
Although some new mothers challenged the restriction against washing and bathing, others maintained the traditional restricted hygiene practices. One participant believed that washing her hair could lead to harmful effects in later life. She stated:

"I didn't wash my hair and bathe; I only cleaned myself with a towel. I have learned that after delivery skin pores will open up and I try to do what I can. I am not young so I need to keep an eye on some taboos."

\section{Social Proscriptions}

The PNC's policies promoted restricted visiting hours, based on the rationale that having many visitors could increase the risk of illness for mothers and babies. During the fieldwork period, visiting hours were restricted after $10 \mathrm{pm}$. Some new mothers indicated that they would have preferred greater visiting hour limitations to improve infection control, and one participant mentioned that she felt reassured that her baby was protected in the communal nursery:

"Before coming to the center, I didn't know that the baby should only be in contact with its father and mother, while others should be isolated and see it through the glass. However, it is good because it can protect the baby from any infection... I find the policy is good for the baby."

In addition to maintaining the taboo of limiting visitors, all participants abstained from sexual intercourse during doing the month. Several reasons were provided for adhering to this restriction. Some participants mentioned that they "felt weakened," "needed rest", that their "wounds had not healed", and that they "did not want to risk infection". All participants mentioned that they would wait until after their postpartum check-up before resuming sexual intercourse to ensure that their health was maximized. This attitude was expressed by one participant:

"I don't feel like having sex because my [episiotomy] wound is not healed yet. I heard from the nurse that I need to wait until the lochia stops and after the postpartum check-up. It is fair enough. No sex for forty-two or fifty-six days."

Although all of the new mothers abstained from sexual intercourse and limited visitors during doing the month, the reasons provided for abstaining were not the traditional ones, such as to avoid misfortune, but were related to health, infection control, and physiological reasons.

\section{DISCUSSION}

\section{Demographic information}

Although the participants of this study were predominantly middle-class women, their participation in doing the month indicates that these women still conceptually adhered to traditional cultural beliefs and practices. The results from this study suggest that doing the month practices may still be widely observed in Taiwanese society, and thus confirm the findings of similar studies that have been conducted in Western countries that have substantial Chinese communities, such as America, Scotland, and Australia (Chen, 2011; Cheung, 1997; Chu, 2005). 
Traditionally, providing care for a daughter-in-law is a mother-in-law's social obligation to foster motherand daughter-in-law kinship bonds. However, $80 \%$ of new mothers who participated in this study were living in nuclear family units, and were no longer able to rely on their mothers-in-law to assist them during doing the month at home. Our findings suggest that the relationship between mother- and daughter-in-law has become more negotiable and is no longer consistent with the traditional rules, which required new mothers to merely listen, and never question the elder's orders. Some participants indicated that the mothers-in-law's help was sometimes unwelcome, mainly because it is difficult to express disagreement with one's mother-in-law. These results are consistent with previous studies that identified a growing trend of the new mothers' own mothers and midwives being the key caregivers during doing the month (Holroyd et al., 2011b; Hung et al., 2010).

\section{Dietary Practices}

At the PNC, traditional dietary practices prescribing well-balanced and nutritious meals were maintained, because postpartum women felt that they needed nutrient-rich foods to maintain health and produce breast milk for their babies. Meats such as pig's liver that are high in iron and lipid-soluble vitamins, especially vitamin A (a nutrient often deficient during the postpartum and lactating period) (Bodnar et al., 2005) were regularly consumed. Several participants consumed a variety of dishes, including beef, pork knuckles, fish, poultry, eggs, vegetables, fruit, and milk, which is a finding that confirms the results (Holroyd et al., 2011b; Wang et al., 2008) that Chinese postpartum women follow traditional dietary proscriptions to promote their physical recovery following childbirth. Traditional doing the month practices are consistent with World Health Organization (WHO)(WHO, 1998) guidelines that recommend an increase of $10 \%$ to $20 \%$ of caloric intake while lactating. Participants in this study generally preferred to base their diet on contemporary understandings of well-balanced postpartum nutrition that were consistent with traditional dietary practices.

\section{Dietary Practices Regarding "Yang" Food for Lactation and Health}

Traditional dietary practices in the "yang" category have high energy values and are rich in nutrients. The traditional recommendation is to eat meat and especially to drink gravy, which is believed to nourish the body by warming it and increasing breast milk production. There is a conflict between traditional dietary practices and contemporary scientific evidence related to physiology and contemporary nutritional standards that maintain that high-fat foods increase the risk of heart disease, obesity, and stroke (Micha \& Mozaffarian, 2010). Reducing the consumption of high-fat dishes is consistent with contemporary scientific knowledge and is practiced to reduce the risk of chronic diseases. Therefore, dietary practices at the PNC have been modified according to contemporary scientific knowledge. The new mothers considered proper nutrition and the consumption of high-calorie meals vital for lactation and physical recovery. Traditional dietary practices related to "yang" foods at the PNC have been modified based on contemporary scientific knowledge.

\section{Dietary Practices Regarding "Cold" Foods}

Traditional postpartum dietary practices advocate that cold drinks, vegetables, fruit, and soy bean products that are considered "cold" foods, should be avoided. However, new mothers who follow this 
diet may lack some nutritional elements, because the prohibition of vegetables, fruit and milk in the traditional diet contradicts contemporary health care knowledge. Liu et al (2006) argued that traditional postpartum diets were unlikely to provide sufficient nutrients to meet the daily vitamin $C$ and dietary fiber requirements for postpartum women. Contemporary health care practices encourage women to eat a well-balanced diet from all food categories during this period, and our study found that women are modifying the traditional diet to include vegetables and fruit. Their diet was generally based on contemporary nutritional principles that advocate the consumption of meat, milk, vegetables, and fruit, indicating a marked departure from the traditional diet (WHO, 1998). Therefore, traditional postpartum dietary practices, such as the avoidance of eating vegetables and fruit, were challenged and adjusted according to contemporary scientific knowledge.

\section{Restricted Hygiene Practices}

These results have demonstrated that rigid adherence to the prohibition of washing and bathing was difficult to maintain and contradicts contemporary health-care approaches and notions of comfort. Contemporary facilities maintain a comfortable environment for new mothers, indicating that doing the month at the PNC is integrated with contemporary scientific knowledge and awareness of comfort. These results are consistent with the findings of previous studies (Chen, 2011; Holroyd et al., 2011a; Liu et al., 2012) that demonstrated that interpretations of the ritual are flexible and that its practice now incorporates contemporary knowledge. Traditional doing the month hygiene practices have been challenged and modified according to contemporary scientific knowledge, which has affected restrictions of hygiene practices.

\section{Restricted Physical Activities}

Traditional postpartum practices encourage new mothers to stay indoors and avoid all types of physical activity. In modern health care, instead of restrictions, postpartum women are encouraged to gradually re-incorporate exercise in their daily routine because it may help them to regain adequate muscle tone, increase muscle strength, and reduce weight (Ko et al., 2013). It is also recommended for alleviating negative physical and psychological symptoms (Claesson et al., 2013). Traditional postpartum practices related to limiting physical activities potentially cause health problems such as constipation, osteoporosis, and excessive weight gain (Bray \& Bouchard, 2004). Our results indicate that the traditional prescriptions, such as limiting physical activities, and engaging in a few outdoor activities were commonly followed by new mothers, despite being challenged by contemporary scientific evidence.

\section{Restricted Social Engagement}

Social restrictions that include limitations on visiting others or entertaining visitors, as well as abstaining from sexual activities during the postpartum period, translate as a period of ritual impurity (Posmontier \& Horowitz, 2004). This study's results indicate that traditional doing the month practices that require new mothers to stay indoors and abstain from sexual activities were maintained. However, the reasons provided for adhering to these rules have developed to incorporate contemporary knowledge. The participants emphasized that they maintained the practices to restore their physical 
health and prevent episiotomy wound infection, rather than for superstitious reasons, such as preventing misfortune for their partners. These results are consistent with previous studies (Holroyd et al., 2011b; Raven et al., 2007) that have reported that postpartum women still adhere to the traditional prescribed behaviors to stay indoors and abstain from sexual intercourse. Additionally, health care professionals in Taiwan typically suggest abstaining from sexual activities for approximately 6 weeks until the postpartum physical check-ups are completed to ensure that the episiotomy wound is sufficiently healed (Nadeau, 2012). Thus, the practice of not having sex during doing the month was adhered to, but the rationale for it has changed.

\section{Limitations of the study}

The sample size in this study was appropriate for the nature of qualitative study. However, the limitation of a single setting and Chinese-speaking women in Taipei are recognized. Participants in this study were healthy, highly educated, middle or upper class women, and had healthy newborns. Therefore, the findings may not be transferable to larger culturally diverse populations where postpartum practices may be conducted differently. Further, this study focused on the practices themselves, and further research could explore the role and activities of health professionals with regard to doing the month ritual practices.

\section{CONCLUSIONS}

Contemporary scientific knowledge and social changes have influenced the traditional ritual practices of doing the month, including the dietary restrictions, hygiene practices, physical activities, and other social proscriptions. Although our participants performed the practices at a PNC, no participant followed all the traditional prescribed rules and restrictions. Many participants were able to modify traditional practices independently. Therefore, midwives should also be able to integrate the positive aspects of doing the month in contemporary evidence-based health-care practice. Additional research is required to determine whether postpartum nursing centers can improve parents' childcare competence and maternal role development.

\section{ACKNOWLEDGMENT}

The authors thank the new mothers who participated in this project. Without them, this work would not have been possible.

\section{REFERENCES}

Bernard HR. Research methods in anthropology: Qualitative and quantitative approaches Lanham: AltaMira Press, 2011.

Bodnar LM, Cogswell ME, Mcdonald T. Have we forgotten the significance of postpartum iron deficiency? Am J Obstet Genecol 2005; 193: 36-44.

Bray GA, Bouchard C. Handbook of obesity: Clinical applications. New York: Maercel Dekker Press, 2004. 
Chen SS: Zuo Yue Zi sitting the month in Taiwan: Implications for intergenerational relations.

Department of Anthropology, Cleveland: Case Western Reserve University, 2011.

Chen Y. Chinese values, health and nursing Journal of Advanced Nursing 2001; 36: 270-275.

Cheung NF. Chinese zuo yuezi (sitting in for the first month of the postpnatal period) in Scotland. Midwifery 1997; 13: 55-65.

Chu CM. Postnatal experience and health needs of Chinese migrant women in Brisbane, Australia. Ethnicity and Health 2005; 10: 33-56.

Claesson IM, Klein S, Sydsjö G, Josefsson A. Physical activity and psychological well-being in obese pregnant and postpartum women attending a weight-gain restriction programme. Midwifery 2013 doi: 10.1016/j.midw.2012.11.006.

Creswell JW. Research design: Qualitative, quantitative, and mixed method. London: Sage Press, 2003.

Denzin NK. Interpretive ethnography: Ethnographic practices for the 21st century. London: Sage Press, 1997.

Forster DA, McLachlan HL, Rayner J, Yelland J, Gold L, Rayner S. The early postnatal period: Exploring women's views, expectations and experiences of care using focus groups in Victoria, Australia. BMC Pregnancy and Childbirth 2008; 8: 27-38.

Gaoa LL, Sunb K, Chan SWC. Social support and parenting self-efficacy among Chinese women in the perinatal period. Midwifery 2013; doi: 10.1016/j.midw.2013.06.007.

Hammersley M, Atkinson P. Ethnography: Principles in practice. New York: Routledge Press, 2007.

Holroyd E, Lopez V, Chan SWC. Negotiating "doing the month": An ethnographic study examing the postnatal practices of two generations of Chinese women. Nurs Health Sci 2011; 13: 47-52.

Howell EA. Lack of patient preparation for the postpartum period and patients' satisfaction with their obstetric clinicians. Obstet Gynecol 2010; 115: 284-289.

Hung $\mathrm{CH}$, Hsiu-Hung Wang, Chang SH, Jian SY, Yang YM. The health status of postpartum immigrant women in Taiwan. J Clin Nurs 2012; 21: 1544-1553.

Hung $\mathrm{CH}$, Yu CY, Liu CF, Stocker J. Maternal satisfaction with postpartum nursing centers. Res Nur Health 2010; 33: 345-354.

Ko YL, Yang CL, Fang CL, Lee MY, Lin PC. Community-based postpartum exercise program. J Clin Nurs 2013; 22: 2122-2131.

Lauderdale J: Childbearing and transcultural nursing care issues. In: Andrews M, Boyle J (eds). Transcultural concepts in nursing care. Philadelphia: Lippincott, 1999; 81-106.

Leininger M. Cultural care diversity and unversality: A theory of nursing. New York: National League for Nursing Press, 1991.

Lincoln YS, Guba EG. Naturalistic inquiry. London: Sage Press 1985.

Liu-Chiang CY. Postpartum worries: An exploration of Taiwanese primiparas who participate in the Chinese ritual of Tso-Yueh-Tzu (doing-the-month). Matern Child Nurs J 1995; 23: 110-122.

Liu N, Mao L, Sun X, Liu L, Chen B, Ding Q. Postpartum practices of puerperal women and their 
influencing factors in three regions of Hubei, China. BMC Public Health 2006; 6: 274-281.

Liu YQ, Maloni JA, Petrini MA. Effect of postpartum practices of doing the month on Chinese women's physical and psychological health. Biol Res Nurs 2012 doi: 10. 1177/1099800412465107.

Martin A, Horowitz C, Balbierz A, Howell EA: Views of women and clinicians on postpartum and recovery. Matern Child Health J 2013; doi: 10.1007 /s10995 -013-1297-7

Martin J. Cultures in organizations: Three perspectives New York: Oxford University Press, 1992.

Maso L. Phenomenology and ethnography. London: Sage Press, 2001.

Micha R, Mozaffarian D. Saturated fat and cardiometabolic risk factors, coronary heart disease, stroke, and diabetes: A fresh look at the evidence. Lipids 2010; 45: 893-905.

Munhall PA. Nursing research: A qualitataive perspective. Sudbury: Jones and Bartlett, 2007.

Nadeau J: The postpartum family: Needs and care. In: Davidson MR, London ML, Ladewig PA (eds). Maternal-newborn nursing \& women's health across the lifespan. London: Pearson Press, 2012.

National Statistics, ROC Taiwan: The list of Baby Friendly Initiative hospitals in Taiwan. Taipei: Executive Yuan, 2013.

Ngai FW, Chan SWC. Stress, maternal role competence, and satisfaction among Chinese women in the perinatal period. Res Nur Health 2012; 35: 30-39.

Ohashi Y, Asanorn M. Transition to early parenthood, and family functioning relationships in Japan: A longitudinal study. Nurs Health Sci 2012; 14: 140-147.

Pillsbury BLK. "Doing the month": Confinement and convalescence of Chinese women after childbirth. Soc Sci Med 1978; 12: 11-22.

Posmontier B, Horowitz JA. Postpartum practices and depression prevalences: Technocentric and ethnokinship cultural perspectives. J Transcult Nurs 2004; 15: 34-43.

Raven JH, Chen Q, Tolhurst RJ, Garner P. Traditional beliefs and practices in the postpartum period in Fujian Province, China: A qualitative study. BMC Pregnancy and Childbirth 2007; 7: 1-11.

Steinberg S. Childbearing research: A transcultural review. Soc Sci Med 1996; 43: 1765-1784.

Strand MA, Perry J, Guo J, Zhao J, Janes C. Doing the month: Rickets and postpartum convalescence in rural China. Midwifery 2009; 25: 588-596.

Wang X, Wang Y, Zanthou S, Wang J, Wang J. A population-based survey of women's traditional postpartum behaviours in Northern China. Midwifery 2008; 24: 238-245.

WHO. Postpartum care of the mother and newborn: A practical guide [Cited June 02 2013]. Available from URL: http://whqlibdoc.who.int/hq/1998/WHO RHTMSM_98.3.pdf. 
Table 1: Interview Guide for the New Mothers

\section{Semi-structured questions}

1. Why did you choose to practice doing the month at a post-natal centre?

2. What has your experience of doing the month been like?

3. Do you strictly follow the practices of doing the month?

4. What do you think is the most important aspect of doing the month?

Table 2: Demographic Characteristics of the Participants

\begin{tabular}{lcc}
\hline & $\mathbf{n}$ & $\%$ \\
\hline Age & 3 & 11 \\
$25-29$ & 17 & 63 \\
$30-34$ & 7 & 26 \\
$35-39$ & & \\
Education & 4 & 15 \\
Junior College & 14 & 52 \\
University & 6 & 22 \\
Masters & 3 & 11 \\
PhD & & \\
Employment & 17 & 63 \\
Yes & 10 & 37 \\
No & & \\
Family Unit & 5 & 81 \\
Living with parents-in-law & 52 \\
Living with husband & 22 \\
\hline
\end{tabular}


Table 3: Doing the month at the post-natal centre: Themes

\begin{tabular}{|c|c|}
\hline Categories & Codes \\
\hline $\begin{array}{l}\text { Reasons for } \\
\text { practicing doing the } \\
\text { month at the PNC }\end{array}$ & $\begin{array}{l}\text { - } \begin{array}{l}\text { Have adequate rest } \\
\text { - } \\
\text { - }\end{array} \text { The baby more relaxed } \\
\text { - } \quad \text { Respect for the traditional ideals of doing the month } \\
\text { - } \quad \text { Learning childcare skills } \\
\text { - } \quad \text { Lack of a helper } \\
\text { - } \quad \text { Avoid "monitoring" by the older generation, } \\
\text { - } \quad \text { Complexity of doing the month procedures } \\
\text { - } \quad \text { The variety of dishes and nutritional balance of doing the month } \\
\text { meals at the MCC. }\end{array}$ \\
\hline Dietary practices & $\begin{array}{l}\text { Maintained } \\
\text { - I don't like sesame oil chicken, but I eat it for my health. } \\
\text { - } \quad \text {..as long as I can follow the traditional way, I just follow it. } \\
\text { - } \quad \text { I keep an eye on eating and take a lot of Chinese herbs. } \\
\text { Modified } \\
\text { - Vegetables and fruit are good for my health, you know... I chose } \\
\text { vegetables such as cauliflower, grapes, strawberries, and apples. } \\
\text { Challenged } \\
\text { - } \quad \text { I won't follow the taboos of doing the month entirely. } \\
\text { - } \quad \text { leat the diet similar to that of normal people. } \\
\text { - We have had good foods every day; my nutrition is good enough. }\end{array}$ \\
\hline $\begin{array}{l}\text { Restricted Hygiene } \\
\text { Practices }\end{array}$ & $\begin{array}{l}\text { Maintained } \\
\text { - I didn't wash my hair and only cleaned it with a towel to prevent the } \\
\text { effects of old age. } \\
\text { - I didn't wash my hair to satisfy the needs of the seniors [in the } \\
\text { family], and let them feel assured. } \\
\text { - My family asked me not to wash and bathe. I'd like to take their } \\
\text { advice. } \\
\text { Modified } \\
\text { - } \text { After washing, using a hair dryer is a good way to do [it] } \\
\text { Challenged } \\
\text { - I didn't follow the taboos of not washing and bathing. } \\
\text { - I felt very uncomfortable before I washed my hair. } \\
\text { - I use boiled water to clean and it [the episiotomy wound] is healing } \\
\text { well and not hurting anymore. }\end{array}$ \\
\hline Restricted Physical & Maintained \\
\hline
\end{tabular}


- I went out and was exposed to winds, and now I have soreness in my neck. I did find it really uncomfortable. I believe postpartum women should not be exposed to the wind.

- The knowledge passed down by ancients is supported by reasons.

- I think to lie in bed this much is not good for health

Modified

- As for the taboo not to go out,...I was tightly bundled to prevent exposure to the wind.

\section{Challenged}

- I came down to walk around three hours after delivery.

\section{Social Restriction Maintained}

- I don't feel like having sex because my [episiotomy] wound is not healed yet.

- I never consider myself unclean. I want no sex life during the doing the month simply because I don't want it,

\section{Challenged}

- There was no restriction for visiting. I like my friends visiting; I want to talk to them.

- I feel bored when I stay here alone.

- Here [the center] is like a camp; it's rather fun! 